\title{
Wittgenstein e a verdade
}

\section{Marcondes Rocha Carvalho ${ }^{1}$}

1 Doutorando em Filosofia da Faculdade de Letras da Universidade do Porto, Portugal.

E-mail: mrcarvalhos@gmail.com Orcid: http://orcid.org/0000-0002-5545-6772

RESUMO: Este trabalho investiga a questão da verdade em Ludwig Wittgenstein (1889-1951). Buscar-seá analisar o artigo Wittgenstein on Truth (2016) de Paul Horwich, confrontando-o com o de Hans-Johann Glock, intitulado Truth in the Tractatus (2006), tendo como objetivo compreender os pressupostos filosóficos centrais das teorias correspondencialista e deflacionária da verdade. Para tanto, na primeira parte, faremos uma caracterização geral dos elementos centrais das teorias substancialista e não-substancialista da verdade; na segunda parte, comentaremos as dificuldades apontadas por Horwich (2016) e Glock (2006) na identificação da concepção de verdade do Tractatus Logico-Philosophicus (1922) enquanto correspondencialista e caracterizaremos, conforme Horwich (2016), os três defeitos da teoria da verdade do primeiro Wittgenstein; na terceira parte, mostraremos que Horwich também se baseia no que Glock denomina de teoria da verdade oficial do Tractatus, bem como as razões pelas quais Horwich (2016) considera ilegítima a introdução do deflacionismo no Tractatus como tentativa de salvar a teoria da verdade tractatiana; na quarta parte, faremos uma breve caracterização da concepção de verdade nas Philosophical Investigations - PI (1953), apontando a sua centralidade na reorientação do pensamento wittgensteiniano. E, por fim, como considerações finais, mostraremos as diferentes conclusões de Horwich (2016) e Glock (2006), assinalando também um aspecto positivo e outro negativo do deflacionismo.

Palavras-chave: verdade, correspondência, deflacionismo.

ABSTRACT: This work investigates the question of the truth in Ludwig Wittgenstein (1889-1951). It will look analyses the papper Wittgenstein on Truth (2016) of Paul Horwich, confronting it with it of Hans-Johann Glock, entitled Truth in the Tractatus (2006), having like aiming to understand the central philosophical presuppositions of the correspondence and deflationary theories of truth. For so much, in the first part, we will do a general characterization of the central elements of the theories substantive and non-substantive of truth; in the second part, we will comment the difficulties pointed by Horwich (2016) and Glock (2006) in the identification of the truth conception in Tractatus Logico-Philosophicus (1922) while correspondence and we characterize, according to Horwich (2016), three defects of the theory of truth of early Wittgenstein; in the third part, we will show that Horwich also is based on what Glock calls of theory of the official truth of the Tractatus, as well as the reasons for which the Tractatus finds Horwich (2016) illegitimate the introduction in the deflationism in like attempt of saving the theory of truth in Tractatus; in the fourth part, we will do a short characterization of the truth conception in Philosophical Investigations - PI (1953), pointing to his centrality in the redirection of the wittgensteinian thought. And, finally, like final considerations, we will show the different conclusions of Horwich (2016) and Glock (2006), marking also a positive aspect and another negative of the deflationary.

Keywords: truth, correspondence, deflationism.

\section{Introdução}

Para analisarmos as intuições wittgensteinianas acerca da verdade, começaremos caracterizando, de forma geral, duas concepções de verdade: o correspondencialismo e o deflacionismo. Há duas classes de 
teorias da verdade: as substancialistas e as não-substancialistas. As primeiras são as teorias clássicas que investigam a verdade enquanto uma propriedade genuína, cujas natureza e essência subjacentes devem ser desvendadas. Por sua vez, as teorias não-substancialistas consideram que a verdade não é uma propriedade genuína, isto é, não se trata de noção metafísica substantiva. ${ }^{1}$

Podemos ilustrar essas teorias por meio das duas formas pelas quais empregamos intuitivamente a palavra "verdade." Por exemplo, quando atribuímos verdade às informações transmitidas pelas nossas sentenças, queremos dizer que esses conteúdos são verdadeiros por possuírem uma propriedade objetiva, que independe de tais sentenças serem formuladas. Por outro lado, emprega-se a palavra "verdade" para expressar a aprovação de uma sentença específica. Se alguém enuncia, por exemplo, que "Lisboa é a capital de Portugal", pode-se expressar a nossa aprovação dizendo "É verdade que Lisboa é a capital de Portugal", ou seja, está-se a fazer uma atribuição de verdade ao que foi enunciado. Embora as sentenças sejam diferentes, o conteúdo cognitivo de ambas é o mesmo. Dessa forma, afirmar uma sentença ou atribuir verdade à mesma não acrescenta nada, não altera o seu valor cognitivo. Então, neste caso, para os defensores da abordagem deflacionista, "é verdadeiro" não é um predicado legítimo, nem designa uma propriedade de natureza substantiva. Não devemos considerar essa expressão como semelhante às expressões "é vermelho" e "têm dois metros de altura", por exemplo.

É preciso explicitar-se mais alguns elementos envolvidos nos exemplos acima. Como se vê, a noção de verdade está sendo concebida como uma relação entre um elemento ao qual o predicado é atribuído - os portadores da verdade (truth-bearer) - e um outro elemento que faz com que essa atribuição seja verdadeira os criadores da verdade (truth-maker). Não obstante, proposições, crenças, sentenças ou enunciados são alguns dos candidatos a truth-bearers e fatos, estados de coisas ou o mundo, como os truth-makers. Daí, nas teorias substancialistas em geral, os conteúdos transmitidos pelas sentenças, a proposição, seriam o truthbearer e a propriedade objetiva real (por exemplo, um fato), o truth-maker. Por exemplo, na abordagem substancialista mais amplamente conhecida, a verdade é uma relação de correspondência entre os truth-bearers (proposições) e os truth-maker (a realidade). Estão aí presentes três elementos que devem ser explicados: truthbearer, uma relação de correspondência e truth-maker. Por sua vez, nas abordagens não-substancialistas, como a verdade não é considerada um predicado genuíno, não teríamos um truth-maker substancialista. Sendo assim, as definições de verdade seguem o esquema geral $(G)$ a seguir:

(G) X é verdadeiro se e somente se ... (Engel 2002: 14)

Mais precisamente, as teorias clássicas insistem na redução da verdade a certas noções fundamentais e substantivas e, em $(\mathrm{G})$, X está para o truth-bearer e o espaço em branco do lado direito deve ser preenchido pelo truth-maker, o qual especificaria uma característica fundamental, como: correspondência com os fatos, coerência com um conjunto $\mathrm{S}$ de sentenças, etc. Sendo assim, as teorias correspondencialistas da verdade (TC) têm como formulação geral:

(TC) X é verdadeiro se e somente se corresponde aos fatos. ${ }^{2}$

Para Glock (2006), há pelo menos duas principais versões das teorias correspondencialistas da ver-

1 Cf.: Engel 2002; Horwich 2001; Bar-On \& Simmons 2008 e Horwich 2010.

2 As teorias substancialistas produzem definições como: " $\mathrm{x}$ is true iff $\mathrm{x}$ has such-and-such characteristic." (Horwich 2010: 06) 
dade: as da correspondência objectual (CO) e as da correspondência factual (CF). A abordagem tradicional exemplifica a primeira versão, já que compreende o truth-maker como um objeto. A relação de correspondência se estabelece entre o predicado e o sujeito da sentença. Por exemplo, na sentença "Sócrates é grego", o predicado "é grego" deve ser adequar, ou corresponder, ao seu sujeito, no caso "Sócrates." Eis, conforme Glock, a formulação geral da concepção objectual tradicional de correspondência:

(CO) A sentença $s$ é verdadeira se e somente se $s$ corresponde ao seu objeto. ${ }^{3}$

Mas (CO) tem um defeito grave pois, para cada sentença, é preciso especificar o sujeito e há sentenças em que não há um sujeito genuíno, como: "Ninguém é perfeito" ou "It is raining". Esse problema será resolvido pela versão em que a correspondência é tratada por meio da noção de fato. Sendo assim, a relação de correspondência se estabelece entre a sentença e o fato ou realidade enquanto truth-maker. Não obstante, para Glock (2006), pode-se identificar três variantes do correspondencialismo factual. Em termos mais aproximados, o fato enquanto truth-maker pode gerar três definições distintas de verdade: na primeira, os fatos correspondentes são plurais (fatos - CF1); na segunda, o fato corresponde é singular (fato - CF2); e, na terceira, a existência de um fato correspondente é afirmada (CF3). Vejamos como Glock (2006) as formula:

(CF1) A sentença sé verdadeira se e somente se corresponde aos fatos.

(CF2) A sentença sé verdadeira se e somente se corresponde ao fato que $s$ expressa.

(CF3) A sentença $s$ é verdadeira se e somente se existe um fato ao qual $s$ corresponde. ${ }^{4}$

Essas três definições de verdade resolvem a falha de (CO); porém, para cada uma delas, pelo menos uma séria dificuldade pode ser colocada. A saber, (CF1) tem menos conteúdo do que (CO), já que a equivalência entre "correspondem aos fatos" e "é verdadeiro" não é esclarecedora. (CF2), por sua vez, relaciona uma sentença verdadeira e o fato que ela expressa, estabelecendo assim uma correspondência do fato expresso pela sentença e o seu correlato na realidade, porém uma sentença falsa não expressa fato algum. Então, não haverá nenhum elemento que demonstre a não satisfação da relação de correspondência, ou adequação. Enquanto (CF3), por fim, evitaria todas as dificuldades mencionadas, mas não consegue explicar a correspondência entre uma sentença verdadeira e o fato que a verifica (Glock 2006: 349).

Por outro lado, conforme Engel (2002), para os teóricos deflacionistas, como asserir uma sentença $(\mathrm{X})$ e atribuir verdade à mesma são processos equivalentes, então o espaço em branco em $(\mathrm{G})$ não deve ser preenchido por nenhuma propriedade substantiva, mas sim pela própria sentença $(\mathrm{X})$. E, dessa maneira, o esquema geral das teorias deflacionistas (TD) seria:

(TD) X é verdade se e somente se X.

Frank P. Ramsey (1903-1930) é considerado o precursor da teoria da redundância, uma das variantes do deflacionismo, e Ludwig Wittgenstein (1889-1951) o grande motivador do deflacionismo por defender,

3 Conforme Glock: Object correspondence, OC: Sentence $s$ is true iff $s$ corresponds to its object, 2006: 348.

4 Glock denomina CF1, CF2 e CF3 respectivamente de fact-correspondence plural (FCP), fact-correspondence singular (FCS), fact-correspondence existence (FCE) e assim os formula: Sentence $s$ is true iff it corresponds to the facts $\mathrm{CF} 1$, Sentence $s$ is true iff $s$ corresponds to the fact $s$ expresses (CF2) e Sentence $s$ is true iff there is a fact to which s corresponds (CF3), 2006: 349. 
segundo Paul Horwich, as diferentes funções das palavras da nossa linguagem (Horwich 2010: 04). Contudo, Gottlob Frege (1848-1925) já tinha mostrado a chamada transparência do predicado "verdade", demonstrando que afirmar a verdade de uma proposição $(p)$, ou seja, dizer que “ " $p$ ' é verdadeiro", ou "É verdade que $p$ ” e afirmar “ $p$ ” significa a mesma coisa (Engel 2002: 42). Portanto, (TD) é uma versão do princípio da equivalência $(\mathrm{E})$ :

(E) É verdade que $p$ se e somente se $p$.

E, conforme Frege, na leitura de (E), no lado esquerdo, o $p$ designa o conteúdo da sentença “ $p$ ”, o truth-bearer. Não obstante, uma outra forma de leitura de (E), também aplicável a (TD), é chamada de concepção da verdade enquanto descitação (disquotation):

(TDD) " $p$ " é verdadeiro se e somente se $p$.

(TDD) é outra variante deflacionista, na qual o truth-bearer são as sentenças, o predicado "verdade" será circunscrito à linguagem, percorrendo as sentenças. As teorias deflacionistas da verdade são as nãosubstancialista e não-clássicas, cuja variante mais radical propõe a eliminação do predicado "verdade". A última proposta deflacionista que comentaremos consiste na concepção minimalista da verdade de Paul Horwich, cujos truth-bearers são as proposições e o predicado "verdade" desempenha apenas a função de dispositivo de asserção e de generalização (Engel 2002: 43). Como afirma Horwich:

Our concept of truth is a device of generalization. That is its function. And it is able to perform that function in virtue of its role within the so-called equivalence schema " $<p>$ is true iff $p$ ". Nothing more is needed; and nothing less will do. It becomes plausible, therefore, that our overall use of the truth predicate is explained ... by our inclination to accept instances of the equivalence schema. (2010:5)

A estratégia subjacente ao minimalismo consiste na ideia de que os enunciados trivialmente especificam as suas condições para serem verdadeiros e, disso, resulta a nossa aceitação intuitiva de instância de equivalências. Por exemplo, "Há vida extraterrestre” se e somente se houver vida extraterrestre, "Tortura é errado" se e somente se torturar for errado. E, conforme Horwich (2010), qualquer atribuição de verdade a uma dada proposição equivale à própria proposição. A inclinação para aceitar a instância da equivalência demonstra que o papel desempenhado pelas nossas atribuições de verdade implica no reconhecimento de que se "Há vida extraterrestre" é verdadeira, então há vida extraterrestre; e, se "Torturar é errado" é verdadeira, então torturar é errado, e assim por diante. A função da verdade, portanto, consiste em permitir a generalização dessa conjunção infinita. Em outras palavras, a nossa inclinação para aceitar a instância da equivalência pressupõe o nosso desejo de acreditar no que é verdadeiro e apenas no que é verdadeiro (Horwich 2010: 6), pois se "Há vida extraterrestre" é verdadeira, então há vida extraterrestre. E, se há vida extraterrestre, então "Há vida extraterrestre" é verdadeira.

\section{Tractatus: representação, correspondência e verdade}

Começaremos essa secção com as perguntas: qual é a teoria da verdade proposta por Wittgenstein no Tractatus? E, como coloca Paul Horwich (2016), o que parece saltar das primeiras páginas do Tractatus é "algo como" uma teoria correspondencial da verdade. Hans-Johann Glock (2006) também afirma que o 
Tractatus tem sido considerado o locus classicus desse tipo de teoria. Então, o que será um truth-bearer e um truth-marker no Tractatus? Ser verdadeiro está sendo concebido como uma relação de correspondência entre truth-bearers (sentenças, proposições, crenças) e truth-makers (fatos, realidade)? De imediato, pode-se afirmar que, no Tractatus, a determinação desses dois elementos não é muito clara e que, talvez, isto seja o calcanhar de Aquiles da sua suposta teoria da verdade como correspondência. Nesta secção, veremos porque, conforme Horwich, temos que fazer certas ressalvas ao classificar de correspondencial a teoria da verdade do Tractatus.

Cumpre ressaltar que "verdadeiro" e "falso", embora possamos falar de sentenças, está sendo aplicado, na maioria das vezes, para falar sobre aquilo que as sentenças expressam (a proposição de que $p$ ). Essa distinção não é muito clara devido à própria terminologia do Tractatus. Dessa forma, para formularmos a concepção de verdade presente no Tractatus, faremos, seguindo Horwich (2016), a tradução da terminologia tractatiana para a nossa atualmente. Wittgenstein, no Tractatus s chama de "signo proposicional", "proposição" e "o sentido da proposição" o que respectivamente poderíamos entender por "sentença não-interpretada", "sentença significativa" e "o fato possível que é representado por uma sentença significativa." (Horwich 2016: 96). Portanto, conforme Horwich (2016), a teoria da verdade do Tractatus diz respeito às sentenças significativas, e não ao que ela representa como sendo o caso e, assim, poderia ser formulada: " $A$ sentence is true iff (i) it represents a certain possible fact; (ii) that possible fact is actual." Ou seja:

(TS) S é uma sentença verdadeira se e somente se $\mathrm{S}$ representa um fato (um fato possível existente). ${ }^{5}$

Portanto, no Tractatus, temos uma teoria sentencial da verdade (TS), que dependerá da noção de sentenças enquanto figurações ou modelos da realidade, da noção de representação figurativa. E, assim, para os nossos propósitos, devemos caracterizar como Wittgenstein concebe essa noção. Nesse momento, seguiremos o esboço da teoria da figuração feito por Horwich (2016), tendo em vista mostrar que a noção de correspondência se aplica mais propriamente ao que Wittgenstein chama de sentido da proposição (o fato possível), e não à sentença verdadeira.

No Tractatus, Wittgenstein afirma que as sentenças são figurações (imagens ou modelos). Uma sentença, então, será capaz de representar algo (que tal e tal é o caso) exatamente do mesmo modo que um mapa ou modelo arquitetônico também é. A representação figurativa pressupõe uma certa disposição dos elementos da figuração, que cada um desses elementos tenha um referente e que o fato atual dos elementos da figuração estarem dispostos ou combinados de uma certa forma deve representar o fato possível de que os seus respectivos referentes estejam também combinados da mesma forma (Horwich 2016: 97). Adaptando o exemplo de Horwich (2016), imaginemos um mapa de Portugal em que "A", "B" e "C" estão respectivamente para as cidades do Porto, de Coimbra e de Lisboa. No mapa, o fato atual de "B" estar entre "A" e "C" deve representar o fato possível de que Coimbra está localizada entre o Porto e Lisboa. Os elementos do mapa, da figuração, e os seus referentes são dispostos ou combinados da mesma forma; a saber, $x$ estar entre $y$ e ₹. Embora inicialmente não pareça, similarmente isso também se dá no caso das sentenças. Por exemplo, para Wittgenstein, uma sentença também é uma imagem, é um fato figurativo o representado por "João ama Maria"; a saber, "João" está à esquerda de "Ama”, que, por sua vez, está à esquerda de "Maria". E o fato representado e o fato possível têm em comum a mesma forma $(a R b)$. Sendo assim, o que é representado consiste numa possibilidade, a de que João mantem a relação amar com Maria,

${ }^{5} \mathrm{Cf} .: \mathrm{S}$ is a true sentence iff S represents a fact (= an actualized possible fact), Horwich 2016: 97. 
ou seja, de que "João ama Maria".

Compartilhar a mesma forma pressupõe, então, uma correspondência entre representação e representado. Daí, a noção de correspondência será central para a concepção da natureza figurativa das sentenças. As sentenças são fatos figurativos que, por sua vez, representam um fato possível. As sentenças enquanto figurações somente podem representar que tal e tal é o caso (um fato possível) se houver uma forma de correspondência entre figuração e afigurado.

A sentença, então, compartilha algo com o que ela representa. O que ambos compartilham é o que Wittgenstein chama de forma de afiguração (Form der Abbildung). Significa que os elementos da sentença e os elementos daquilo que é representado compartilham a mesma multiplicidade lógica. Uma certa combinação de nomes na sentença deve estar pela mesma combinação dos seus respectivos objetos (referentes desses nomes) nos estados de coisas possíveis. Em outros termos, como afirma Glock (2006), no Tractatus, a representação exige um isomorfismo lógico, a representação e o representado têm a mesma forma, sentenças elementares e fatos possíveis compartilham a sua forma de afiguração.

Dessa forma, sob uma perspectiva, pode-se considerar que, no Tractatus, as dificuldades das teorias correspondencialistas baseadas na noção de fato (como as CF1, CF2 e CF3) são solucionadas. E, sobretudo, a teoria da figuração fornece uma explicação para a até então silenciada noção de correspondência por meio do isomorfismo entre linguagem e mundo (Glock 2006: 352). É exatamente o apelo ao isomorfismo enquanto aquilo que torna uma sentença verdadeira que, como afirma Glock (2006), constitui o principal equívoco das interpretações que identificam no Tractatus, ${ }^{6}$ uma teoria da verdade como correspondência, pois o isomorfismo entre sentenças elementares e fatos possíveis não é condição suficiente para a determinação da verdade das sentenças, mas sim condição para a determinação do sentido, pois entendemos uma sentença se sabemos o que ela representa, um fato possível (Glock 2006, 347: 351). Como podemos verificar nos aforismos 2.161, 2.17 e 2.22, para que a sentença possa ser uma figuração do mundo, deve haver algo de idêntico entre sentença e estados de coisas possíveis. E o que têm em comum é a sua forma de afiguração, para representar correta ou falsamente. Então, a sentença será uma afiguração de estados de coisas independente de sua verdade ou falsidade.

Afirmar que uma sentença é verdadeira se e somente se for isomórfica com o que representa não satisfaz as condições para que possamos determinar a verdade de uma sentença, dado que, conforme a teoria da figuração, todas as sentenças significativas, não somente as verdadeiras, representam o mundo. Ou, conforme Glock (2006):

The relation of isomorphism explains not what it is for a sentence to be true, but what it is for a sentence to say something. That the configuration of objects in a possible situation corresponds to that of the simple signs in the sentential sign means not that the latter is true, but that it depicts the former. The Tractatus links the sense of a sentence to the conditions under which it is true. To understand a sentence is to know what is the case if it is true (4.024). Isomorphism guarantees that a sentence does have truth-conditions, not that these truth-conditions are fulfilled. (2006: 353).

Eis a principal dificuldade, para Glock (2006), das interpretações que buscam identificar uma teoria correspondencialista da verdade no Tractatus. E, conforme (TS), o termo correspondência aplica-se devido à concepção de sentença como figuração, devido à teoria representacional aí presente.

\footnotetext{
${ }^{6}$ Leia-se: "This explanation - the picture theory - appeals to an isomorphism: a situation and any sentence depicting it must share a logical form, a logico-mathematical multiplicity. Unfortunately, this marks the point at which standard correspondence interpretations go astray." (Glock 2006: 352).
} 
Vejamos agora as ressalvas que Horwich (2016) faz quanto à aplicabilidade do rótulo "correspondência" sobre a concepção de verdade do Tractatus. Segundo Horwich (2016), a proposta de Wittgenstein no Tractatus consiste numa teoria sentencial da verdade de dois níveis, e só o primeiro envolve a noção de correspondência. A teoria da figuração se aplica apenas às sentenças elementares e, assim, correspondência se aplica apenas a tais sentenças, já que como Wittgenstein diz, "My fundamental thought is the 'logical constants' do not represent" (4.0312). Dessa forma, para ponderar-se sobre a verdade ou falsidade de uma sentença molecular, é necessário saber a verdade ou falsidade das sentenças elementares que a compõem. E, por sua vez, saber o que é o caso se uma sentença elementar for verdadeira, é saber o que ela representa, que combinação de objetos a torna verdadeira. Torna-se evidente que a noção de correspondência enquanto isomorfismo entre sentença e fatos possíveis entra em cena apenas no caso das sentenças elementares. As sentenças moleculares terão a sua verdade determinada em função das sentenças elementares. A segunda ressalva de Horwich (2016) diz respeito à possibilidade de afirmarmos que Wittgenstein, no Tractatus, não tem nenhuma teoria "correspondencial" da verdade para aquilo que as sentenças expressam (proposições). Como podemos observar:

One conclusion we might reach is that Wittgenstein does not have, and cannot have, any theory of truth of that sort. That is because the only kind of thing he countenances that resembles what are now called "propositions" are possible facts, and possible facts are not the sorts of things that it makes sense to speak of as "true or false". Rather, such things can only be "actual or nonactual" (that is, "actual or merely possible").

Horwich (2016) tem consciência que essa alegação poderia ser respondida por meio da interpretação segundo a qual "A proposição de que ké fé verdadeira" e "O fato possível de que ké $f$ é atual” são equivalentes e, assim, "fatos possíveis" seriam identificados com "proposições". Mas, ainda segundo Horwich (2016), "we would conclude that Wittgenstein is, after all, implicitly committed to a view of the notion that we call "propositional truth" - the view of it that coincides with what he explicitly says about possible-fact actuality." (2016: 99). Contudo, não é pacífica essa equivalência entre ser verdadeiro e ser atual.

Tendo em vista essas dificuldades, Horwich (2016) afirma que, independente da escolha interpretativa a ser feita, a teoria tractatiana sentencial da verdade consiste em afirmar que quando as coisas significam (quando há a correlação entre nomes e objetos), as sentenças podem ser verdadeiras e têm a qualidade especial de ser atual, ou existir, ou concordar com a realidade. Nas palavras de Horwich (2016):

But whichever choice is made here, we can see that the core of Wittgenstein's proposal about sentential truth is the idea that
only when the things meant (or expressed, or represented) have a certain special quality (-being actual, or being factual,
or obtaining, or existing, or agreeing with reality, ...-) can the sentences with those meanings be true. Thus one might
well suppose that the character of this special quality will be crucial in judging whether his overall view can justly be called
"a correspondence theory of truth". (2016:99).

A concepção de verdade sentencial tractatiana depende do conceito de atualidade (ou obtaining) não explicado por Wittgenstein. Dessa forma, conclui Horwich, "we are given is not really a correspondence theory of truth but rather a limited correspondence theory of representation plus a primitivist non-theory of when the represented entities are facts." (2016: 99).

Em outros termos, Horwich (2016) aponta os três principais defeitos da forma como Wittgenstein, no Tractatus, conceitua a verdade. O primeiro é a caracterização da verdade através de uma noção não explicada, a de fato. Não adianta equacionar "fato" com "ser atual", ou "ser o caso" (obtains), ou "existir" ou "concordar com a realidade", caso não seja explicado nenhum desses termos. O segundo consiste na difícil 
distinção entre fatos atuais e fatos meramente possíveis. Ou seja, "the fact is a certain arrangement of certain entities; but the merely possible fact is exactly that arrangement of exactly those entities. So how could there exist any merely possible facts? Would not all possible facts have to be actual?" (Horwich 2016: 102). O terceiro diz respeito à utilização de uma outra noção, ou relação, não explicado no Tractatus: a relação de referência entre palavras e coisas. (2016: 102).

\section{Um sopro deflacionista na teoria da verdade oficial do Tractatus}

Neste momento, cumpre fazermos uma caracterização do que Glock (2006) considera como a teoria da verdade oficial do Tractatus para sabermos se Horwich (2016) também parte da mesma caracterização. Abordaremos, então, conforme Glock (2006), a teoria da verdade oficial do Tractatus e veremos porquê, para Horwich, introduzir o deflacionismo no Tractatus não seria legítimo. Para Glock (2006), a teoria da verdade oficial do Tractatus é uma obtainment theory of truth (TO) como assim a formula:

(TO) A sentença $s$ é verdadeira se e somente se o estado de coisa $s$ representado existe (obtains). ${ }^{7}$

(TO) combina a teoria da figuração e a ideia de concordar ou não com a realidade para que possamos determinar a verdade de uma sentença. Como vemos nos aforismos:

2.21 A picture agrees with reality or fails to agree; it is correct or incorrect, true or false.

2.222 The agreement or disagreement of its sense with reality constitutes its truth or falsity.

2.223 In order to tell whether a picture is true or false we must compare it with reality.

4.21 The simplest kind of proposition, an elementary proposition, asserts the existence of a state of affairs.

4.25 If an elementary proposition is true, the state of affairs exists: if an elementary proposition is false, the state of affairs does not exist. (Wittgenstein, 2001).

Em outros termos, conforme o Tractatus, o sentido de uma proposição consiste na representação de um fato possível (4.031). O sentido da proposição estabelece a forma como a proposição se relaciona com a realidade, representando estados de coisas possíveis. A relação entre a proposição e a realidade consiste na possibilidade representada pelo seu sentido (2.222). E o que uma proposição elementar expressa é a existência (Bestehen) de um estado de coisas (4.21). Portanto, a determinação da verdade de uma proposição elementar representa a existência de um estado de coisas, a falsidade representaria a não existência (4.25 e 4.031).

Para Glock (2006), (TO) deve ser a base de qualquer interpretação do Tractatus e podemos verificar que a proposta de Horwich (TS) está de acordo com (TO), visto que (TS) estabelece que uma sentença é verdadeira se e somente se representa um fato (um fato possível atual). As noções de representação e existência (obtains) de um estado de coisas de (TO) estão presentes também em (TS). Portanto, Glock (2006) e Horwich (2016) não divergem a respeito dessa base interpretativa e, se (TO) deve consistir numa teoria correspondencialista ou deflacionista da verdade, eis a questão que eles se propõem a responder. Para Glock (2006), muitos dos problemas da teoria da verdade do Tractatus seriam resolvidos se adotássemos uma proposta interpretativa que resumisse os elementos correspondencialista, deflacionário e semântico. Como podemos ver:

the obtainment theory can be seen as a synthesis of correspondence, semantic and deflationary theories. It does justice to the

\footnotetext{
7 Cf:: A obtainment theory of truth é assim apresentada: "Sentence s is true iff the state of affairs s depicts obtains."(Glock, 2006: 356).
} 
idea that whether a sentence is true depends solely on what is the case. And it combines a (loosely speaking) semantic explanation of the relation between a sentence and what it depicts or says with a (loosely speaking) deflationary account of the agreement between what the sentence says and what obtains or is the case if it is true. (Glock, 2006: 347).

Não obstante, a teoria da verdade oficial do Tractatus (TO ou TS) tem os defeitos apontados por Horwich e, mesmo a tentativa de remediá-los através da introdução de intuições deflacionárias, teria como consequência, conforme Horwich (2016), o colapso do Tractatus. Isto significa que a proposta acima de Glock, embora possa nos ajudar a compreender a questão da relação entre linguagem e mundo no primeiro Wittgenstein (Glock, 2006: 346), deve ser questionada quanto à legitimidade da introdução dos elementos deflacionistas.

Nesse momento, mostraremos, conforme Horwich (2016), como a introdução do deflacionismo salvaria a teoria da verdade do Tractatus, mas não seria compatível com o Tractatus como um todo. A pressuposição dos conceitos não explicados de atualidade (obtainment) e referência (relação entre nomes e objetos), bem como a questão de como fatos meramente possíveis podem existir, são as falhas centrais da teoria da verdade do Tractatus (formulada conforme TO ou TS). Tais falhas, conforme Horwich (2016), podem ser corrigidas com a introdução de elementos deflacionários. A saber, a qualidade especial que estabeleceria a distinção entre fatos atuais e meramente possíveis deveria ser abandonada, colocando em seu lugar um esquema que especificaria as condições de atualidade (CA). E Horwich assim sugere: (CA) - O fato possível de que $p$ é atual se e somente se p.

Como afirma Horwich (2016), o equívoco foi pressupor uma qualidade de natureza intrínseca que distinguisse fato e proposição. Ao adotarmos (CA), também estaríamos resolvendo, desmistificando, a noção de fato. Isto porque as condições para que a proposição seja verdadeira serão dadas pelo esquema da equivalência (E). Como Horwich (2016) deixa claro, a sua sugestão deflacionista não interpreta (E) como fazem os defensores da teoria da verdade como identidade, o esquema da equivalência deve ser interpretado como fixando o conceito de verdade e, assim, definindo "fato" como "proposição verdadeira."

E, ainda, a misteriosa relação de referência entre nomes e objetos deve ser substituída pela concepção de significado das palavras como uso, o que permitiria Wittgenstein "to explicate and demystify reference via a pair of schemata along the lines of " $n$ " refers to $x \equiv n$ is identical to $x$ (for names); and "f" is true of $x \equiv f(x)$ (for predicates)."

Horwich (2016) também assinala que a nossa compreensão do lado direito desses esquemas não pressupõe o conhecimento do que é especificado do lado esquerdo. Como podemos ver, o que está pressuposto na introdução de elementos deflacionários acerca da verdade é a sua associação com o deflacionismo referencial e com a noção de significado como uso. E isso não é compatível com o Tractatus.

\title{
0 deflacionismo da verdade nas Philosophical Investigation
}

\author{
Como afirma Horwich:
}

as we have seen, deflationism about truth goes hand-in-hand with deflationism about reference; and deflationism about

\footnotetext{
8 Horwich não interpreta como Jennifer Hornsby e afirma na nota de rodapé 12: she too maintains that true propositions are facts. But her idea is that this equation will define "true" in terms of "fact"-where the latter, roughly in the spirit of the Tractatus, is taken either to be a primitive or to be defined as a "combination of objects and properties". But my deflationary suggestion goes in the opposite explanatory direction-that we rely on the trivial Equivalence Schema to fix the concept of TRUTH, and then proceed to define "fact" as "true proposition" (2016: 03).

9 Cf.: Idem.
} 
reference goes hand-in-hand with a use-theoretic view of meaning. But Wittgenstein's later commitment to 'meaning as use' is the principal basis for his recognizing his earlier "grave mistakes". (2016: 104).

Dessa forma, para Horwich (2016), os problemas da teoria da verdade do Tractatus somente podem ser corrigidos, ou serão corrigidos nas Philosophical Investigations (PI), juntamente com uma reorientação filosófica geral. Ou ainda:

So my conclusion is that Wittgenstein's magnum opus, Philosophical Investigations, is a great advance on the Tractatus, his brash, brilliant, initial attempt at a radically anti-theoretical philosophy. And this progress is almost entirely due to the way in which his new view of truth improves on the old one. (Horwich, 2016: 104).

Entretanto, apesar das poucas evidências textuais das PI quanto à questão da verdade, Horwich (2016) a coloca no centro das principais mudanças do pensamento de Wittgenstein, sendo um aperfeiçoamento da sua filosofia anti-teorética. Cumpre fazermos uma breve incursão nas PI, mostrando o que Wittgenstein sustentou explicitamente sobre a questão da verdade.

Nas PI, na secção 136, podemos encontrar:

At bottom, giving "This is how things are" as the general form of propositions is the same as giving the explanation: a proposition is whatever can be true or false. For instead of "This is how things are", I could just as well have said "Suchand-such is true". (Or again, "Such-and-such is false".) But ' $p$ ' is true $=p \mid$ ' $p$ ' is false = not-p. And to say that a proposition is whatever can be true or false amounts to saying: we call something a proposition if in our language we apply the calculus of truth functions to it. (2009: 58).

Essa é a evidência textual que permite, embora haja quem discorde (Bartunek, 2017), concluir que a noção de verdade aí presente associa-se mais proximamente com o deflacionismo. Contudo, como o próprio Horwich reconhece, não é fácil saber qual das variantes atuais do deflacionismo Wittgenstein subescreveria (Horwich, 2016: 100). Não obstante, nas PI a associação entre deflacionismo acerca da verdade, deflacionismo referencial e a noção de significado como uso é natural. Nesta secção, apenas comentaremos, conforme Horwich (2016), o deflacionismo referencial: "Going hand-in-hand with deflationism about truth is the idea that our notions of predicative and nominal REFERENCE are fixed by the schemata: $\boldsymbol{f}()$ is true of $x=f(x) ; \boldsymbol{n}$ refers to $x$ $=n$ is $x . "$ (Horwich, 2016: 100). Neste esquema o seu lado direito - $f(x)$ e $n$ é $x$-é fixado através do uso dessas expressões, assim como também utilizamos sentenças, sem necessitarmos de conhecer os referentes. O lado esquerdo, o que especificaria os referentes, não precisa ser conhecido para que possamos utilizar as expressões. Nas palavras de Horwich (2016):

specify what a given concept applies to, but only given prior possession of that concept (which is deployed on the right-hand side of the relevant equations) — a possession that, on pain of circularity, cannot derive from knowing the concept's reference but must instead consist in mastery of its use. (2016: 100).

A circularidade aconteceria se para compreender o lado direito fosse pressuposto o conhecimento do lado esquerdo, dos referentes. Daí, retornaríamos ao ponto em que nossa tentativa de explicar a verdade pressuporia noções não esclarecidas (fato, referência nome-objeto). Portanto, os deflacionismos predicativos e nominais têm o mesmo efeito que o veritativo, já que analogamente as noções de referência e de verdade mostrar-se-iam superficiais e vazias. Por exemplo, afirmar que ' $p$ 'é verdadeiro, que 'f( )' é verdadeiro e que ' $n$ ' se 
refere a x não são especificações que determinam a nossa compressão desses conceitos, mas formas superficiais que não são determinantes para o uso, já que é no contexto de uso que se constituem enquanto significativos.

\section{Considerações finais}

Neste trabalho buscamos construir um debate entre Horwich (2016) e Glock (2006), procurando demonstrar que ambos identificam as principais dificuldades em qualificar a teoria da verdade do Tractatus como correspondencialista e tendo em vista, sobretudo, compreender duas das principais teorias da verdade e lançar luz sobre as intuições wittgensteinianas acerca da questão no Tractatus e nas PI, uma vez que é possível localizar essas diferentes intuições na sua primeira obra, ou ainda, como sugere Bartunek (2017), traçar uma continuidade da noção de bipolaridade nessa última, que consiste naquilo que Wittgenstein chamou de sua teoria no Notebooks 14-16. ${ }^{10}$

Contudo, cumpre sublinhar que não reconstruímos o debate mencionado exaurindo as questões presentes nos artigos analisados, mas buscando identificar pequenas convergências (TS e TO). Isto não significa que ambos cheguem a resultados similares, pois enquanto Glock (2006) não considera legítimo recusar o caráter correspondencialista da teoria da verdade do Tractatus, Horwich (2006), por sua vez, sugere que nem mesmo uma teoria da verdade o Tractatus teria; melhor seria identificar uma limitada teoria correspondencial da representação. Nosso objetivo não é, pois, tomar parte nessa disputa, mas antes compreender as dificuldades envolvidas nas abordagens correspondencialista e deflacionária da verdade.

A principal dificuldade das interpretações correspondencialistas do Tractatus consiste, conforme Glock (2006), no papel que a tese do isomorfismo desempenhará para a determinação da verdade. Entretanto, essa tese tem uma função na determinação do sentido das proposições, e não na determinação da verdade. A proposta interpretativa de Glock (2006) pode ser vista como uma solução que busca unificar diferentes intuições wittgensteininas sobre a questão da verdade, desde a ideia de verdade e falsidade como concordância e discordância com a realidade até aquela segundo a qual "uma proposição é verdadeira se as coisas estão como, por meio dela, dizemos que estão." (Wittgenstein, 1994: 4.062) A primeira tem claramente um viés correspondencialista e a segunda, deflacionário. E, ainda, para Glock, não podemos negar o primeiro viés sob pena de termos que negar que as abordagens de George E. Moore (1873-1958) e Bertrand Russell (1872-1970) não são correspondencialista, já que Wittgenstein, no Tractatus, teve como modelo a abordagem desses dois. Sendo assim, a teoria da verdade oficial do Tractatus (TS ou TO), para Glock (2006), baseia-se em (CF) e resolve os problemas de (CO), (CF1) e (CF2), podendo também ser formulada conforme (CF3). E mais, o Tractatus consegue dar um conteúdo para a até então silenciada noção de correspondência. Horwich (2016), por sua vez, considera que a alegação segundo a qual negar o caráter correspondencialista da verdade, no Tractatus, a qual implicaria negar também esse mesmo caráter das abordagens de Moore e Russell, não tem fundamento, dado que as abordagens destes são dissemelhantes e não-análogas às do Tractatus. ${ }^{11}$

$10 \mathrm{Cf} .:$ "Every proposition is essentially true-false. Thus a proposition has two poles (corresponding to case its truth and case of its falsity). We call this the sense of a proposition. The meaning of a proposition is the fact which actually corresponds to it. The chief characteristic of my theory is: $p$ has the same as not-p. (1969: 94)

11 Horwich afirma na nota de rodapé 7 que Glock “goes on to allow that it is still appropriate to call Wittgenstein's view "a correspondence theory" since the similar view of truth that is proposed by both Moore and Russell around 1912 is standardly regarded as paradigmatic of such theories. But it is worth noting a relevant dis-similarity. As we have seen, the foundation of Wittgenstein's account is his distinction between those possible facts that are actual and those that are not; and actuality is not a correspondence notion. But there is no analogous distinction in the account 
Ficam, assim, evidentes as conclusões incongruentes de Glock (2006) e Horwich (2016).

Quanto à nossa busca pela compreensão das intuições filosóficas presentes nas teorias da verdade substancialistas e não-substancialistas, pode-se dizer que as abordagens deflacionárias da verdade têm um compromisso metafísico em comum associado com uma pretensão de desmistificar a verdade: a verdade não é uma propriedade genuína e substantiva. ${ }^{12}$ Talvez essa tese metafísica negativa atraia quem não está disposto a admitir uma metafísica inflacionada como a pressuposta nas teorias da verdade como correspondência. Contudo, embora essa desmistificação pareça vantajosa, há uma certa insatisfação com o deflacionismo, pois, como sublinha Deviit (2001), os enfoques de ambas as teorias citadas são distintos: enquanto uma investiga a natureza substantiva da verdade (metafísica da verdade), a outra investiga e descreve a função da palavra "verdade". Dito de outro modo, não há dúvidas de que as abordagens correspondencialistas decepcionam aqueles que buscam uma metafísica mínima, ou melhor, que possa ser logicamente coerente; e as deflacionistas também decepcionam pela insatisfação causada: parece que não se chegou ainda com precisão ao cerne conceitual do que se pretende exprimir quando do emprego da palavra "verdade".

\section{Referências}

Bartunek, N. (2017). "Truth in the Investigations". Synthese: https://doi.org/10.1007/s11229017-1643-1.

Bar-On, D.; Simmons, K. (2008). “Deflationism” In: E. Lepore and B. C. Smith (ed.), The Oxford Handbook of Philosophy of Language. Oxford: Clarendon Press.

Deviit, M. (2001). “The Metaphysics of Truth” In: M. Lynch (ed.). The Nature of Truth. Cambridge, MA: MIT Press.

Engel, P. (2002). Truth. London: Acumen Publishing.

Glock, H.-J. (2006). “Truth in the Tractatus”. Synthese: 148/2, 345-368.

Horwich, P. G. (2001). “Teorias da verdade” In: J. Branquinho and D. Murcho (org.), Enciclopédia de termos lógico-filosóficos, Lisboa, Gradiva, 730-736.

. (2010). Truth-Meaning-Reality. Oxford: Clarendon Press.

. (2016). “Wittgenstein on Truth”. Argumenta: 2/1, 95-105.

Wittgenstein, L. (2001). Tractatus Logico-Philosophicus. London: Routledge Classics, 1922.

. (1994). Tractatus Logico-Philosophicus. Tradução Luiz Henrique Lopes dos Santos. São

Paulo: Edusp, 1922b.

. (2009). Philosophical Investigations. Oxford: Blackwell, 1953.

. (1969). Notebooks 1914-1916. Blackwell, Oxford, 1961.

Artigo recebido em: 02 de dezembro de 2019

Artigo aceito em: 26 de fevereiro de 2020

offered by Russell and Moore. They hold, for reasons we will be examining in section 4, that there can be no such things as false propositions. And this reasoning would lead them to the same skeptical conclusion about merely possible facts." (2016: 103).

12 Esse compromisso é claramente expresso por Bar-On \& Simmons 2008, 610 e a pretensão desmistificadora, podese, encontrar um exemplo em Horwich, 2010: 4. 